Henry Keazor

\title{
Zu zwei Zeichnungen Nicolas Poussins
}

Innerhalb der reichen Literatur, welche sich mit dem CEuvre Nicolas Poussins befaßt, nehmen die seinen Hermen für den Park von Vaux-le-Vicomte gewidmeten Titel einen vergleichsweise schmalen Raum ein ${ }^{\mathrm{I}}$. Dies mag zum einen in der Ausnahmestellung dieser von Nicolas Fouquet bestellten Skulpturen innerhalb des CEuvres von Poussin begründet sein, welches ansonsten nie zu einem solchem Gattungssprung von der Malerei zur Skulptur zwingt; über die eventuelle Scheu vor einer solchen Überschreitung hinaus spiegeln sich in dieser Zurückhaltung jedoch wohl zum anderen auch die großen Probleme wider, vor welche sich eine Untersuchung dieses Themas von Anfang an gestellt sieht. Denn angesichts des Erhaltungszustandes der Skulpturen sowie der wenigen über sie berichtenden, zumeist eher trüben oder einander widersprechenden Quellen erscheint es als eine schier unlösbare Aufgabe, definitive Aussagen zum Entwurf ${ }^{2}$, zur Authentizität ${ }^{3}$ und Attribution ${ }^{4}$ der einzelnen Figuren zu treffen. Selbst ein kürzlich von Katharina Krause erstmals mit den Hermen in Beziehung gebrachtes Transportin-

${ }^{\mathrm{I}}$ Die Literatur hierzu ist tatsächlich mehr als überschaubar - vgl. Denio, E. H.: Nicolas Poussins Leben und Werk, Leipzig 1898, 95f.; Coural, J.: »Les Termes de Poussin à Versailles«, in: Revue des Arts X, 1960, 67-74; Wild, D.: »Les Termes de Nicolas Poussin dans le Parc de Versailles", in: Revue de la Société Suisse des Amis de Versailles, November 1960, 19-22; Blunt, A.: The Paintings of Nicolas Poussin - A Critical Catalogue, London 1966, 148-157; Bershad, D. L.: »Domenico Guidi and Nicolas Poussin «, in: The Burlington Magazine, CXIII, I971, 544-547; Wild, D., Nicolas Poussin-Leben, Werk, Exkurse, Zürich 1980, Bd. 2, I7if.: Souchal, F.: French Sculptors of the Seventeenth and Eighteenth Century. The Reign of Louis XIV., Bd. 2, 1981, 193, 208 und Bd. 3, 1987, 289f. sowie in jüngster Zeit Krause, K.: »Li varii Genii de' fiori e de' frutti della terrac Poussins Hermen und andere Skulpturen in französischen Gärten des 17. Jahrhunderts «, in: Die Gartenkunst VI, Heft I, 1994, 42-67.

${ }^{2}$ Während Bellori in seinen »Vite " berichtet, Poussin habe originalgroße Terracottamodelle als Arbeitsvorla- ventar vermag zwar endlich Aufschluß über den genauen Zeitpunkt ihrer Versendung von Rom nach Frankreich zu geben', nicht jedoch das dringliche Problem der Identifizierung der einzelnen Hermen zu lösen ${ }^{6}$, an welche sich die Beantwortung weiterer Fragen hinsichtlich ihrer Authentizität und Ikonographie ${ }^{7}$ knüpfen könnte.

Diesbezüglich erforderliche neue Quellen können an dieser Stelle nicht präsentiert werden; da jedoch auch hinsichtlich der Entwurfspraxis noch immer eine gewisse Unsicherheit besteht, soll hier näher auf ein Zeichenblatt Poussins eingegangen werden, das von Zeit zu Zeit immer wieder einmal Fragen aufwirft, wenn es als eventuelle Entwurfszeichnung für nie ausgeführte bzw. verschollene Hermen gehandelt wird.

Während Monique Lavallée und Doris Wild ${ }^{8}$ dieses Blatt (Abb. I) aus dem Musée de Besançon stets als Studien für die verschollenen Hermen des »Winters « und des »Sommers « lasen, schloß Anthony Blunt die Zeichnung unter Verweis auf ihre Datierung sowie gewisse motivische Details aus dem Kontext des Hermen-Auftrages aus?. Tat-

ge für die ausführenden Bildhauer geschaffen (vgl. Bellori, G. P.: Le Vite de'pittori, scultori et architetti moderni..., Rom 1672, 436), zitiert Bershad in seinem Aufsatz $197 \mathrm{I}$ (Anm. I), 547 eine Notiz Carlo Cartaris aus dem Jahre 1683, welche eine Aussage des römischen Bildhauers Domenico Guidi wiedergibt, der, als einer der an den Hermen beteiligten Bildhauer (vgl. Anm. 4), Bellori widerspricht: Poussin habe lediglich kleine Wachsmodelle geliefert.

${ }^{3}$ Von den heute im Park zu Versailles aufgestellten sechzehn zusammengehörigen Hermen wurden schon Anfang des I8. Jahrhunderts von der Sommer-Ceres sowie dem Winter überliefert, daß sie nicht auf Poussin zurückgehen (vgl. Thomassin, S.: Recueil des statues, groupes, fontaines, termes, vases etc. $d u$ Cbâteau et Parc de Versailles, Paris 1694, Tafel 193: "Cérés«, zu der angemerkt wird, daß sie einWerk J. B. Théodons sei; dessen zweiter Beitrag zur Hermengruppe, der Winter, wird von Thomassin, Tafel rge irrtumlich Pierre Le Gros zugeschrieben - ein Fehler, den bereits Montaiglon und Guiffrey I 888 im zweiten Band ihrer Ausgabe der Cor- 
respondance des directeurs de l'Académie de France à Rome avec les surintendants de Bâtiments, 99 und ro6 korrigieren). Coural I960 (Anm. I), 73f. hält von den vierzehn übrigen noch in Frage stehenden Hermen lediglich neun für authentisch, während er den Bacchus, die Ceres/Abundantia, die Frühlings-Flora im Quinconces du Nord, den Moissoneur und die Bacchante als später hinzugekommene und nicht auf Pous$\sin$ zurückgehende Figuren ausscheidet. Wild 1960 (Anm. I), 20 und 1980, 172 eliminiert lediglich die ersten drei der von Coural ausgeschiedenen Skulpturen, während Blunt 1966 (Anm. I), I54-I57 sämtliche vierzehn fraglichen Figuren auf Poussin zurückführt, bei den Hermen von Bacchus und Moissoneur jedoch nur die Köpfe als authentisch und auf restaurierte bzw. erneuerte Rümpfe gesetzt akzeptiert. Souchal 198I (Anm. I). Bd. 2, 193 und 207 schließt durch auf Briefquellen gestützte Zuschreibungen der Bacchantin und der Frühlings-Flora an Pierre Laviron bzw. Lacroix indirekt zwei Figuren aus (die seinerzeit von Henri Puvis de Chavanne in den Zeitschriften Revue de l'art ancien et moderne LXIII, 1933, I4I-I44 sowie LXVIII, 1935, 93-95 und Beaux-Arts, No. 108 und 109, 1935, initiierte und heute lediglich noch mit Amüsement zu verfolgende Grundsatzdebatte um die Authentizität der Hermen hat damit endgültig nur noch historischen Wert bekommen - Puvis de Chavanne hatte versucht, den Nachweis zu erbringen, daß keine der Hermen etwas mit Poussin zu tun habe; die Frühlings-Flora hatte er Jean Baptiste Tuby I. zugeschrieben). Krause 1994 (Anm. I) übernimmt die Ergebnisse von Souchal und kombiniert sie mit den Beobachtungen Blunts zu Bacchus und Moissoneur.

${ }_{4}^{4}$ Bershad 197I (Anm. I), 544f. kann drei der Hermen (Pan, Pallas und Faunus) aufgrund der oben (Anm. 2) angeführten Notiz Cartaris dem römischen Bildhauer Domenico Guidi zuschreiben. Zu den Zuschreibungen Souchals an Laviron und Lacroix vgl. Anm. 3. Krause 1994 (Anm. I) versucht Zuschreibungen des Bacchus, der Liberalitas oder der Pomona an den römischen Bildhauer Orfeo Boselli, angeregt durch dessen Aussage in seinen Osservazioni della scoltura antica, er habe "doi termini figurati per la Francia« hergestellt. Es würde allerdings etwas verwundern, wenn Boselli sich mit diesem Verweis tatsächlich auf die hier in Rede stehenden Hermen bezöge, ohne dabei (wie auch Guidi) den Namen Poussins zu erwähnen.

${ }^{5}$ Krause 1994 (Anm. I): das Inventar ist auf den 24. Mai 1657 datiert.

${ }^{6}$ Die in dem Inventar (Anm. 5) erwähnten Namen »minerva«, "Pomona«, "satiro«, "Ercole con barba«, "donna vestita moderna u und "Bacco « lassen sich problemlos einigen der Hermen zuordnen; der Text spricht jedoch auch zweimal von "fauna sowie von je einem »autunno « und »Vertunno «, deren Identifikation unter den Poussin-Figuren strittig bleiben muß.

${ }^{7}$ Bellori beschreibt in seinen "Vite" (Anm. 2), 436 eine heute nicht mehr zu identifizierende Ceres sowie einen »Bacco con le spicche, e l'uve «, jenen Attributen also, welche - wie Wild I980 (Anm. I), Bd. 2, 172 ganz richtig

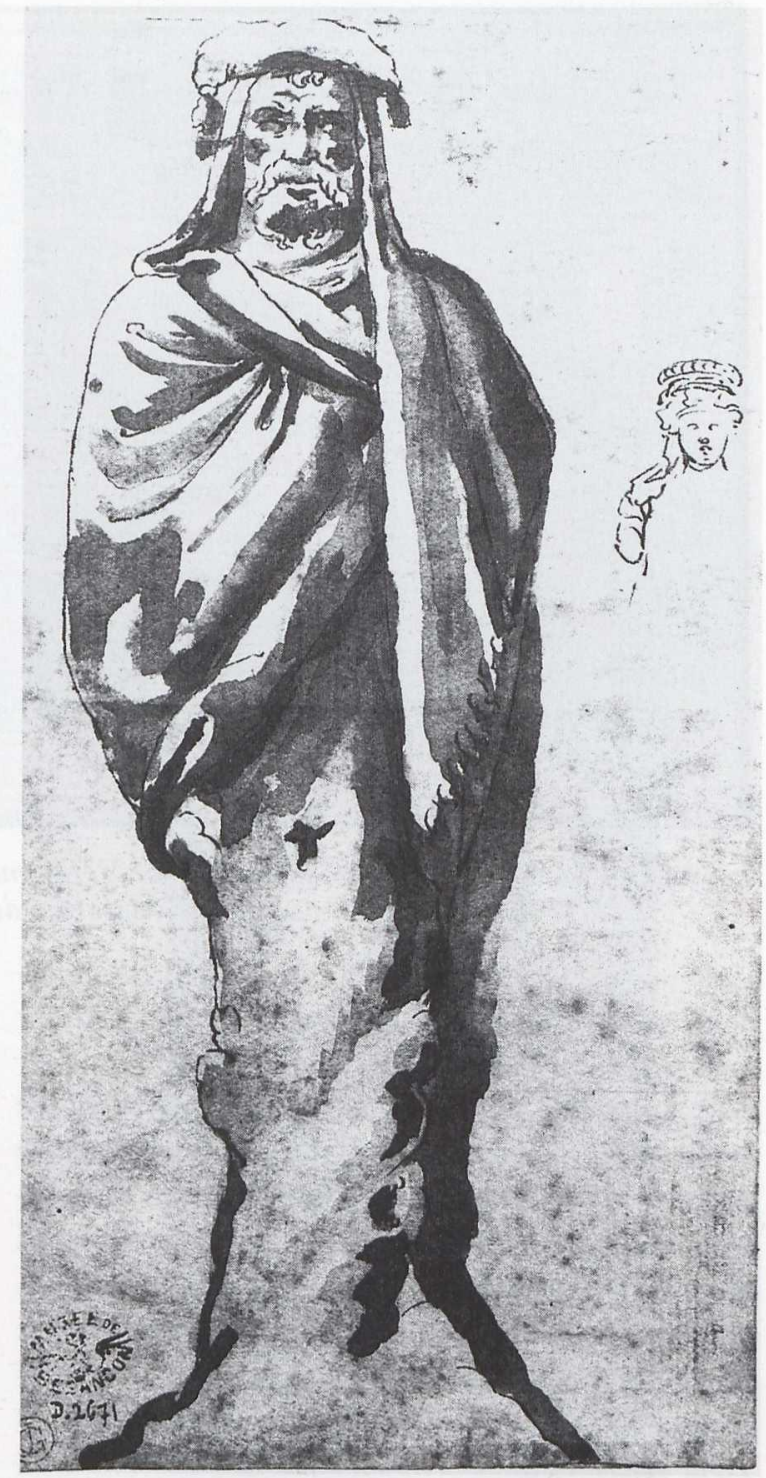

I. Nicolas Poussin, Männliche Karyatide nach einem verlorenen Fassadengemälde Polidoro da Caravaggios und Maturino da Firenzes am Palazzo Gaddi in Rom,

Besançon, Musée des Beaux Arts, Inv. No. 2671

anmerkt - heute nur noch an der Herme der Bacchantin von der Hand Lavirons zu beobachten sind.

${ }^{8}$ Die Meinung von Lavallée im Ausstellungskatalog Dessins français du XVII. siècle, Besançon $1953, \mathrm{II}$, No. 8 sowie zitiert bei Coural 1960 (Anm. I), 74, Anm. 13; zur Interpretation von Wild vgl. Wild 1960 (Anm. I), 2 of. und $1980, \mathrm{Bd} .2,17 \mathrm{I}$.

${ }^{9}$ Blunt, A./Friedländer, W.: The Drawings of Nicolas Poussin, Vol. IV, London 1963, 25, No. 246. Blunt äußerte eine ähnliche Ansicht bereits in dem Kommentar des 


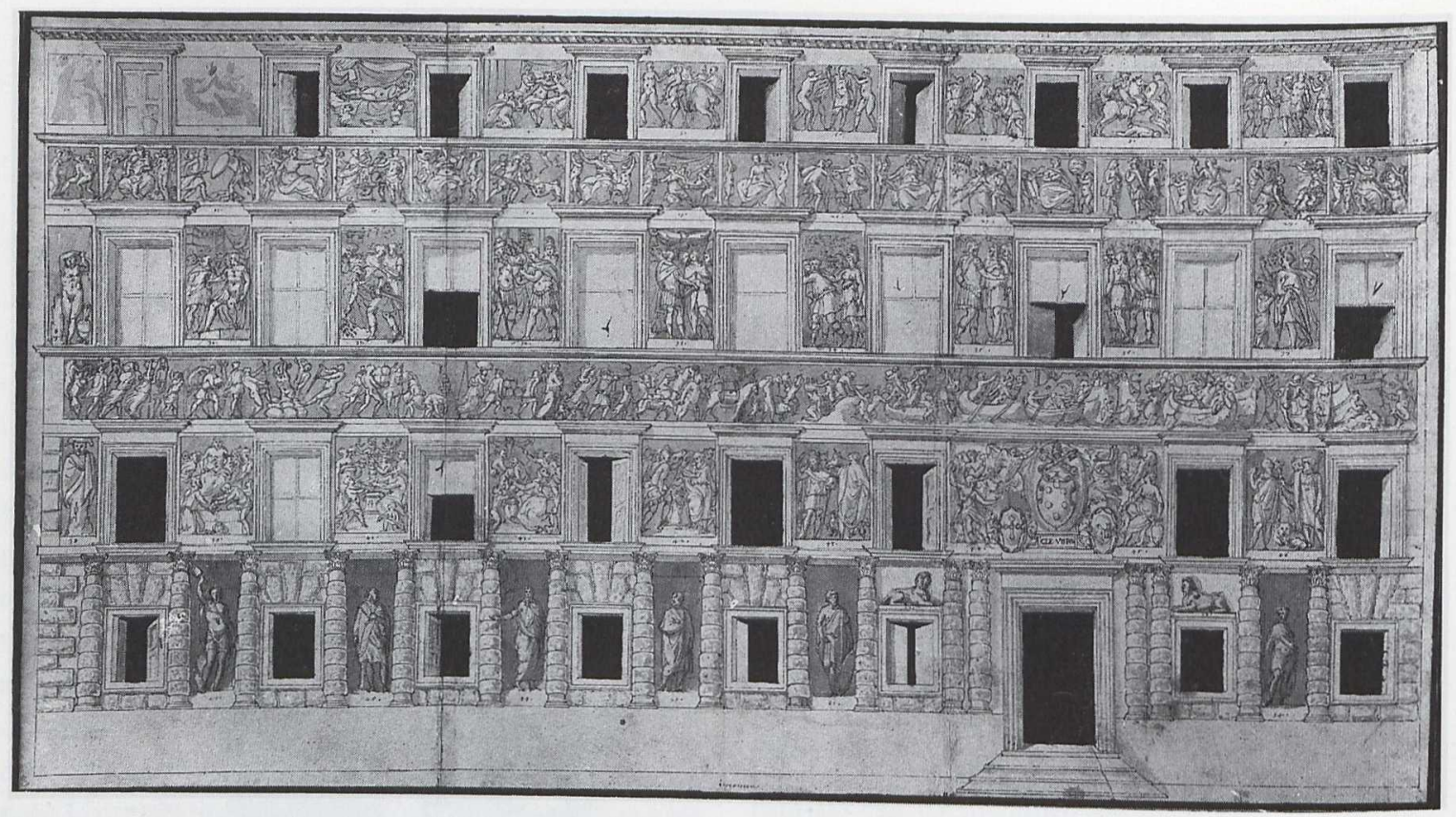

2. Anonymer Künstler des I6. Jahrhunderts, Verlorene Fassadendekoration des Palazzo Gaddi in Rom durch Polidoro da Caravaggio und Maturino da Firenze, Wien, Albertina, Inv. No. 15.462

sächlich erscheint eine Datierung des Blattes in die für den Hermen-Auftrag entscheidenden Jahre um 1655/57 nach stilkritischen Maßstäben unmöglich, vergleicht man es mit den Entwürfen und Skizzen Poussins zu Gemälden, welche relativ zuverlässig in diese Zeit datiert werden können ${ }^{\mathrm{T}}$ : hier jener zittrig-gewellte Strichduktus, wie er typisch für Poussins Zeichenstil ab Mitte der vierziger Jahre ${ }^{\mathrm{II}}$ - die Formen eher füllig umreißt und

Ausstellungskataloges The Age of Lowis XIV, Winter Exhibition der Royal Academy of Arts, London 1958, Bd. I, III, No. 264. Die Meinungen Lavallées und Blunts werden einander gegenübergestellt in den Ausstellungskatalogen Nicolas Poussin et son temps, Rouen 1961, 8I, No. 179 und Französische Zeichnungen aus dem Museum in Besançon, Hamburg 1969, No. 47.

${ }^{10}$ Als stilkritische Eckpfeiler können hierbei z. B. die Zeichnungen zu den Gemälden "Die heilige Familie mit Badebecken und sechs Putten" von I65I (früher Chatsworth, Derbyshire; heute Pasadena, Kalifornien: Norton Simon Found/J. Paul Getty Museum, Malibu; Zeichnung in Chantilly, Musée Condé - vgl. dazu Blunt, A./Friedländer, W.: The Drawings of Nicolas Poussin, Vol. I, London 1939, 27, No. 53) sowie »Achill einkreist als genau bezeichnet - dort, bei der Besançon-Zeichnung hingegen, sparsames Konturieren mit flüssig und sicher geführten Linien; hier eine die Schatten nur grob abtastende Lavierung dort hingegen ein reich differenziertes Modellieren der Formen bis in die Schatten hinein. Es ist Blunt daher nur zuzustimmen, wenn er das Besançon-Blatt in die Jahre um 1635 datiert $^{\mathrm{I} 2}$, in welchen Poussin - wahrscheinlich nach Vorbildern der

unter den Töchtern des Lykomedes « von 1656 (Richmond, Virginia, Museum of Fine Arts; Zeichnung in St. Peterburg, Ermitage - vgl. dazu Blunt, A./Friedländer, W.: The Drawings of Nicolas Poussin, Vol. II, London 1949, 4, No. 106) dienen; zu den Gemälden vgl. Blunt 1966 (Anm. I), No. 58 und No. 127 sowie Wild r980 (Anm. I), Bd. 2, No. 168 und No. 191. In der Ermitage-Zeichnung für das »Achill«Gemälde lehnt Poussin sich in der Knieenden vorne rechts übrigens - bislang offenbar übersehen - sehr eng an die Frauengestalt an, welche in Raphaels "Transfiguration" im Vatikan gleichfalls im Bildvordergrund rechts kniet.

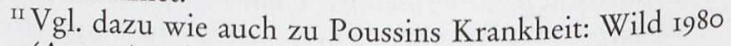
(Anm. I), Bd. I, p. 193. 
Raphael-Schule - mit Feder und Lavierung experimentierte, bis er zu einer für ihn neuen und lange verbindlich bleibenden Technik gefunden hatte ${ }^{\mathrm{I}}$. Deren Eigenart, die sorgfältige Beschreibung und Modellierung der Kleinformen mit Hilfe sehr klarer und starker Schattenwirkungen auf dem Hintergrund vorstrukturierender Feder- bzw. Kreidestriche, läßt sich sehr schön auch an einer nicht zu Ende geführten Zeichnung einer antiken Urne aus dem Metropolitan Museum, New York (Abb. 4) studieren, welche in den gleichen Zeitraum datiert werden $\mathrm{kann}^{\mathrm{I}}$, und zu der an dieser Stelle eine präzisierende Anmerkung eingeschaltet werden soll. James Thompson hat kürzlich einen Vorschlag zur Bestimmung der dargestellten, bislang jedoch nicht identifizierbaren Urne unterbreitet, demzufolge es sich um das Cinerarium des Licinus Crassus Frugi in den Vatikanischen Museen zu Rom handeln könnte ${ }^{15}$. Da jedoch Unterschiede zwischen der von Poussin gezeichneten und der im Vatikan aufbewahrten Urne auffallen (statt von Widder-Köpfen wird letztere an den Ecken von Satyrköpfen gerahmt), vermutet Thompson, daß Poussin » was improvising around his as-yet-unidentified classical prototype $\ll^{\mathrm{I}}$. Eine solche Spekulation erübrigt sich jedoch, wenn man ein anderes Objekt als Modell in Betracht zieht: die aus der Sammlung Ludovisi stammende Ara funeraria im Museo Nazionale Romano ${ }^{17}$ (Abb. 5) stimmt in all ihren Details so exakt mit der von Poussin gezeichneten Antike überein, daß in ihr wohl auch das Vorbild hierzu gesehen werden muß. Zurück zu der Besançon-Zeichnung

${ }^{12}$ Blunt/Friedländer 1963 (Anm. 9), 25, No. 246.

${ }^{13}$ Vgl. dazu Blunt, A.: The Drawings of Poussin, London 1979, $38 \mathrm{ff}$.

${ }^{14}$ Blunt, A./Friedländer, W.: The Drawings of Nicolas Poussin, Vol. V, London 1974, 32 und 33, No. 320 datieren die Zeichnung auf die späten I6zoer Jahre.

is Thompson, James: Nicolas Poussin (= The Metropolitan Museum of Art Bulletin, Winter 1992/93, Vol. L, No. 3), $22 \mathrm{f}$.

${ }^{16}$ Thompson $1992 / 93$ (Anm. 15), 22.

${ }^{17}$ Inv.-No. 8578 ter. Vgl. Giuliano, A. (Hrsg.): Museo Nazionale Romano - Le Sculture; Bd. I, s: I Marmi Ludovisi nel Museo Nazionale Romano, Rom I983, No. 3, 6-10.

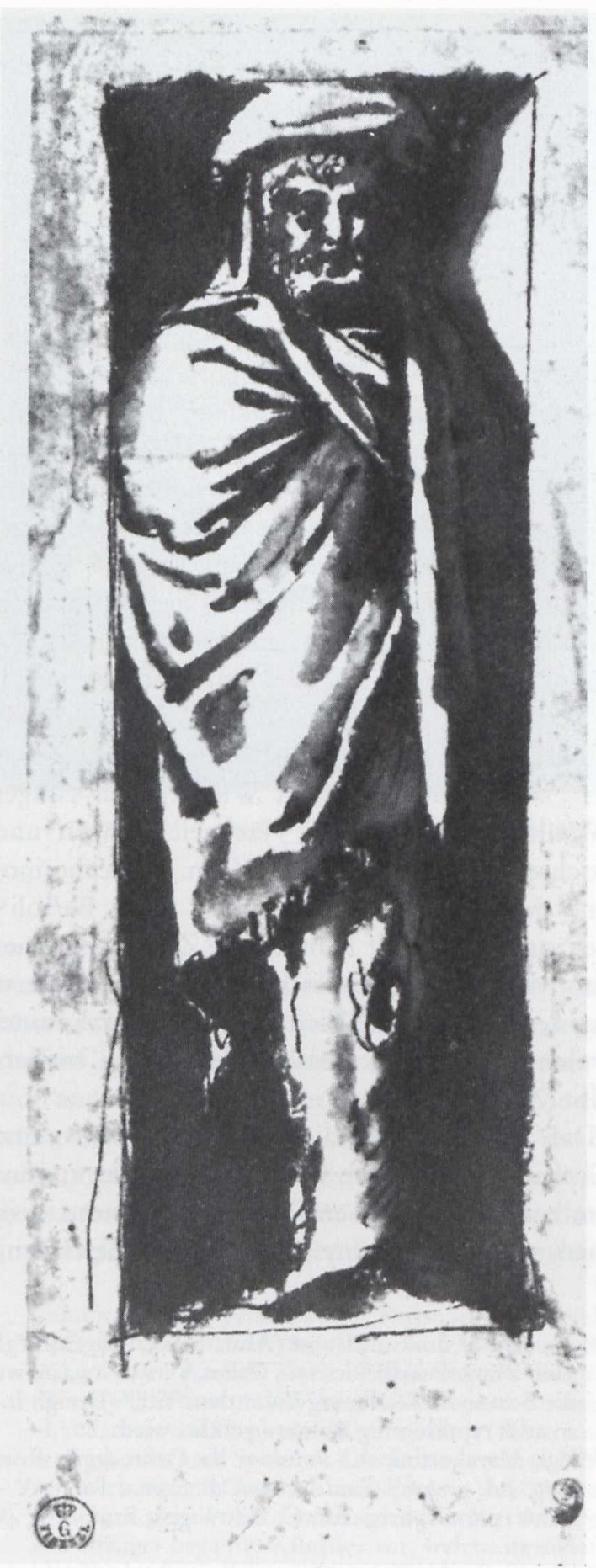

3. Anonymer Künstler des 16. Jahrhunderts, Männliche Karyatide nach einem verlorenen Fassadengemälde Polidoro da Caravaggios und Maturino da Firenzes am Palazzo Gaddi in Rom, Florenz, Gabinetto Disegni e Stampe degli Uffizi, Inv. No. 1290 S 
(Abb. I): gegen ihre Lesart als Hermenstudie führt Blunt auch die Beobachtung ins Feld, daß die dargestellte Figur offenbar darauf angelegt sei, mit ihrem kissenbedeckten Kopf ein Gebälk abzustützen, weshalb er folgert, das Blatt müsse mit einem Architektur-Entwurf zusammenhängen, "which, however, cannot at present be identified $\ll^{18}$.

Wie hier gezeigt werden kann, handelt es sich bei Poussins Zeichnung nun tatsächlich um eine Figur, die einem architektonischen Kontext entstammt: jedoch stellt sie keinen Eigenentwurf Poussins dar, sondern gibt ganz offensichtlich das Detail einer römischen Fassadendekoration des Cinquecento wieder, welche heute zerstört ist.

Diese Fassadendekoration am Palazzo Gaddi (später: Cesi, heute: Camuccini) in der Via della maschera d'oro, ein Gemeinschaftswerk von Polidoro da Caravaggio und Maturino da Firenze, welches auf $1524-1527$ datiert wird ${ }^{19}$, war zunächst nur durch die hymnischen Beschreibungen Vasaris $^{20}$ sowie die teilweisen Wiedergaben einiger Wandfelder in diversen Nachzeichnungen und Stichserien Michele Crecchis (gen. il Lucchesino) und des Poussin-Schülers Pietro Sante Bartoli ${ }^{21}$ bekannt ${ }^{22}$, ehe Rolf Kultzen die Zeichnung eines anonymen Kopisten des späten I6. Jahrhunderts aus der Albertina zu Wien veröffentlichen konnte, welche die gesamte Fassadendekoration wiedergibt (Abb. 2) $)^{23}$.

Daß Poussin Teile dieses in Chiaroscuro und Graffito ausgeführten Gebäudeschmucks zumindestens von den Stichen Crecchis her kannte, weiß man, seitdem Anthony Blunt für eine Zeichnung

${ }^{18}$ The Age of Lonis XIV I958 (Anm. 9), III, No. 264. Vgl. auch Blunt/Friedländer 1963 (Anm. 9), 25, No. 246, wo die Besançon-Zeichnung unter dem Titel "Design for an atlas representing Winter " geführt wird.

19 Vgl. Marabottini, A.: Polidoro da Caravaggio, Rom I969, Bd. I, I25f.; Guide rionali di Roma, Rione V Ponte, parte I, hrsg. von C. Pietrangeli, Rom 1978, 48, No. I5.

${ }^{20}$ Vasari, G.: Le Vite dei più eccellenti Pittori, Scultori e Architetti, I edizione 1550; II edizione 1568; hrsg. von G. Milanesi, Florenz $1878-1885$, Bd. V, I45f.

${ }^{21} \mathrm{Zu}$ diesen Stichen sowie Bartoli als Schüler Poussins vgl. Mazzi, M. C.: "L'incisore perugino Pietro Sante Bartoli«, in: Bolletino della Deputazione di storia patria per l'Umbria, LXX, I, 1973, 21 und 24f.
Poussins in Chantilly die Vorlage eines solchen Stiches nachweisen konnte ${ }^{24}$.

Die Zeichnung aus Besançon jedoch bietet offensichtlich die seltene Gelegenheit, Poussin sogar einmal als Kopisten in actu zu erleben - gibt sie doch ein Detail der Fassadendekoration wieder, welches sich überhaupt nur in wenigen Stichen, dann jedoch lediglich als freie Nachgestaltung fin$\operatorname{det}^{25}$, getreuer aber nur noch in zwei anderen Zeichnungen überliefert wird, von denen Poussins Wiedergabe nicht nur unabhängig zu sein scheint, sondern jene an Genauigkeit sogar noch übertrifft. Auf der oben schon erwähnten Albertina-Zeichnung (Abb. 2) erkennt man im ersten Stock, neben dem linken Eckfenster des Gebäudes, ein schmales Feld, auf welchem die Gestalt einer männlichen Karyatide erscheint. Trotz ihrer geringen Größe ist die Wiedergabe dieser Figur detailreich genug, um ihre enge Verwandtschaft mit der Gestalt der Poussin-Zeichnung erkennen zu lassen: angefangen von dem $z u$ beiden Seiten des vollbärtigen Gesichtes in zwei Zipfeln herunterhängenden Kopfpolster, über den zum Boden hin sich unregelmäßig ausbreitenden, wie grob aus Felsen gehauenen Schaft, bis hin zu den identisch verlaufenden Falten des über Kopf und Oberkörper gelegten bzw. gewickelten Tuches, das in beiden Fällen auf der rechten Körperhälfte in steilen Diagonalen straff von oben und unten auf die linke Achsel zuverläuft, während ein Ende des Stoffes in schrägem Fransensaum über die linke Körperhälfte herabhängt. Das einzige Detail, das die AlbertinaZeichnung mit derjenigen aus Besançon nicht teilt,

${ }^{22}$ Vgl. dazu Pericoli, C. R.: Le case romane con facciate graffite e dipinte, Ausstellungskatalog, Rom I960, 42 f., Marabottini ${ }_{969}$ (Anm. 19), Bd. 2,365-366, No. I4 sowie Ravelli, L.: Polidoro Caldara da Caravaggio, Bergamo 1978, 343-367, No. 611-666.

${ }^{23}$ Kultzen, R.: Rezension zu dem Buch von Pouncey und Gere, "Italian Drawings in the Department of Prints and Drawings in the British Museum: Raphael and his Circle«, London 1963, in: Kunstchronik 16, 1963, 345. Vgl. zu dieser Zeichnung dann auch Marbottini 1969 (Anm. 19), Bd. 2, 366 sowie Ravelli 1978 (Anm. 22), 343, No. 6II.

${ }^{24}$ Blunt/Friedländer 1974 (Anm. 14), 38, No. 337 sowie Blunt 1979 (Anm. I3), I44f. Eine farbige Abbildung der Chantilly-Zeichnung findet sich bei Lévêque, J. J.: La vie et l'œuvre de Nicolas Poussin, Paris 1988, 69. Zu 


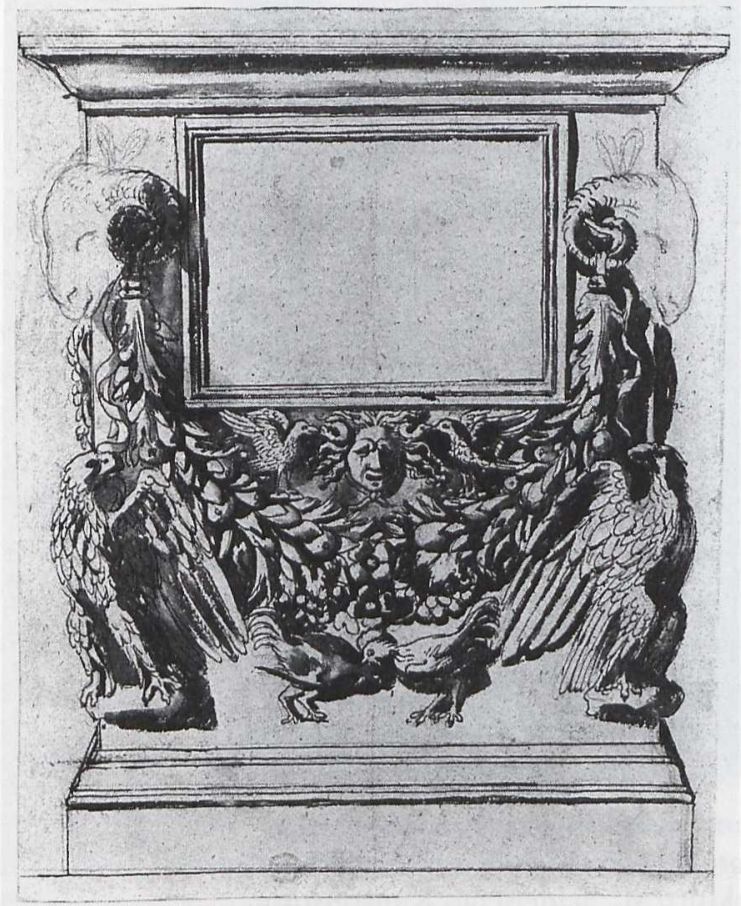

4. Nicolas Poussin, Ara funeraria, New York, Metropolitan Museum, Inv. No. 80.3.583

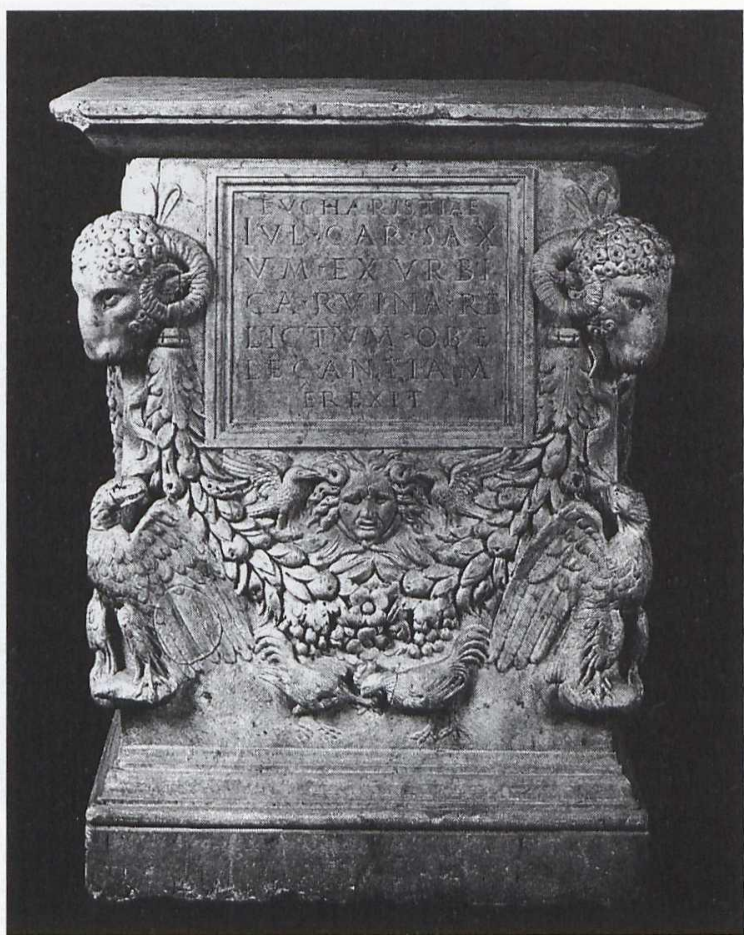

5. Ara funeraria, Rom, Museo Nazionale Romano, Inv. No. 8578 ter ist das bei Poussin zu beobachtende Motiv des sich auf der rechten Schulter in einer weichen schlaufenartigen Falte stauenden Tuches; daß dieses Motiv jedoch nicht seiner Phantasie zuzuschreiben ist, zeigt eine zweite anonyme Zeichnung nach der Gaddi-Fassade aus den Uffizien ${ }^{26}$, welche allein das Feld mit der männlichen Karyatide wiedergibt (Abb. 3). Der Zeichner hat hier zwar

Poussins Polidoro-Verehrung vgl. auch Blunt 1979 (Anm. 13), 6, und 117 sowie Marabottini 1969 (Anm. 19), Bd. I, 3, 55, II5 und I24-Bd. 2, 3ro, No. 51. Poussin sammelte offenbar auch Stiche nach Polidoro - in seinem Nachlaß befanden sich 70 entsprechende Exemplare (vgl. Archives de l'art français, Bd. 6, Paris $1858-$ 1860, 252). Daß er bei Cassiano Dal Pozzo auch Zeichnungen Polidoro da Caravaggios selbst studieren konnte, legen Äußerungen Félibiens nahe, vgl. Félibien, A.: Entretiens sur les vies et sur les ouvrages des plus excellents peintres anciens et modernes, Paris $1696^{2}$, Bd. I (Entr. III), 344f., 36I sowie Bd. 2 (Entr. VIII), 324. $\mathrm{Zu}$ diesen Zeichnungen im Kabinett Dal Pozzos vgl. im übrigen auch die von Émile Magne zitierte Passage aus Deseines Description de la ville de Rome, en faveur des étrangers... aus dem Jahre 1690 in Magne, É., Nicolas auch - wie Poussin - die Technik von Feder und brauner Lavierung angewendet, um den Eindruck der in Chiaroscuro ausgeführten Fassadendekoration annähernd adäquat in das Medium der Zeichnung zu überführen ${ }^{27}$ - doch abgesehen davon und unter Abzug des parallelen Motives der weich gestauten Tuchfalte, erweist sich diese Darstellung im Vergleich zu derjenigen Poussins als ungleich

Poussin-premier peintre du roi, Paris $1928^{2}$, IIs. Ravelli, L.: Polidoro a San Silvestro al Quirinale, Bergamo 1987 , 58 legt einen Einfluß zweier Zeichnungen Polidoros (Wien, Albertina und Windsor Castle) auf Poussins "Landschaft mit Orpheus und Eurydike" (Paris, Louvre) nahe. Richard Symonds überliefert in seinen Notizen nach einem Gespräch mit Poussin dessen Ausspruch, er ziehe Polidoros Fassadengrisaillen den Werken weniger begabter Künstler vor, welche ihre Unfähigkeit durch den Einsatz von Farbe zu kaschieren suchten; vgl. dazu Beal, M.: "Richard Symonds in Italy: his meeting with Nicolas Poussin «, in: The Burlington Magazine CXXVI, 1984, I4I-I44, bes. 143.

${ }^{25} \mathrm{Zu}$ diesen Stichen vgl. Anm. 28.

${ }^{26}$ Ravelli 1978 (Anm. 22), 374, No. 6I4.

${ }_{27}$ Ibidem. 


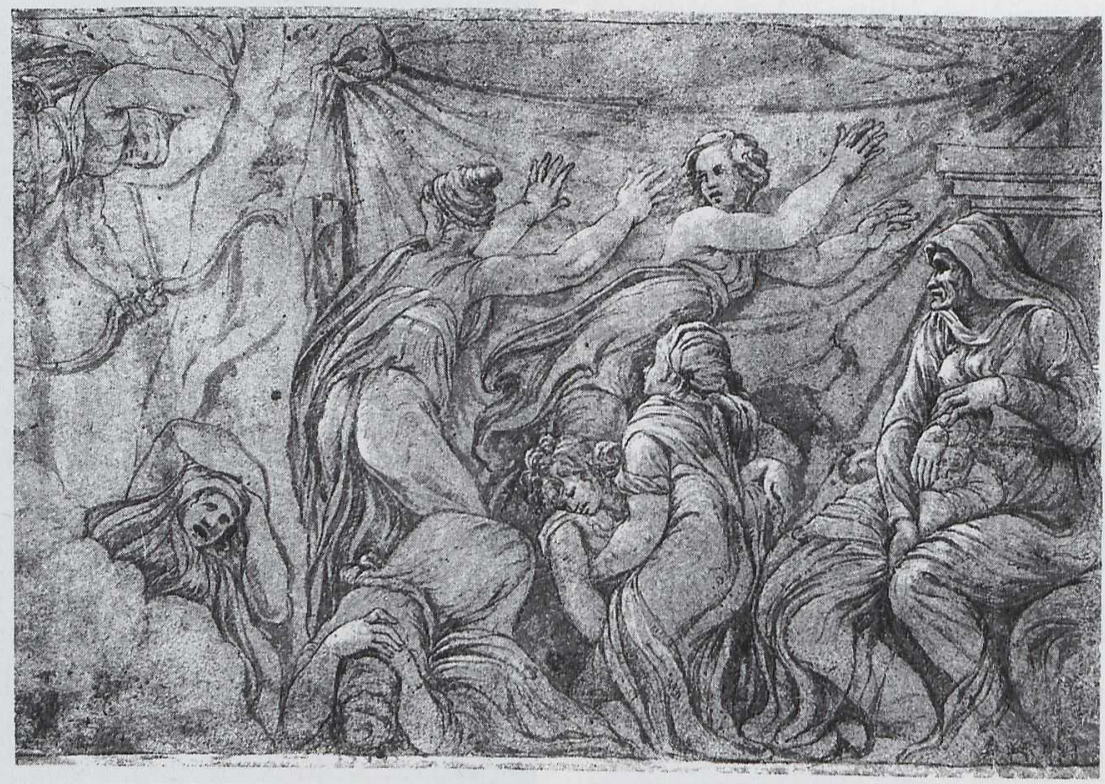

6. Anonymer Künstler des 17. Jhs., Ermordung der Kinder Niobes, nach einem verlorenen Fassadengemälde Polidoro da Caravaggios und Maturino da Firenzes am Palazzo Milesi in Rom, Frankfurt/M., Städelsches Kunstinstitut, Inv. No. 5421

weniger sorgfältig und eher $\operatorname{grob}^{28}$. Während die Albertina-Zeichnung zugunsten ihres Strebens nach überblickverschaffender Vollständigkeit die malerischen Aspekte der Dekoration vernachlässigt, scheint es dem anonymen Zeichner der $\mathrm{Ka}$ ryatide eher um die Wiedergabe von Licht- und Schattenkontrasten, denn die exakte Erfassung einzelner Details gegangen zu sein. Poussin hingegen gibt das Wandbild auf seiner Zeichnung an-

${ }^{28}$ Bartsch, Vol. 26 (neue Ausgabe von 1978, alte Zählung: I4/I) zeigt als No. 304, 302 einen Stich des RaimondiSchülers Agostino (dei Musi) Veneziano (vgl. das Monogramm und die Jahreszahl 1536 auf dem Postament im linken Teil der Darstellung) sowie als No. 304A, 303 dessen spiegelbildliche Kopie durch den Würfelmeister: der Veneziano-Stich zeigt auf der rechten Seite eine Herme, deren Erscheinungsbild - spiegelverkehrt - in seinen wesentlichen Motiven zwar auf die Polidoro-Herme zurückgeht (vgl. Schaft und Drapierung); da diese jedoch im Detail dann gegenüber dem Vorbild so frei variiert und eigenständig ausgestaltet werden, ist dieser Art von Stichen keinerlei Einfluß auf die hier diskutierten Zeichnungen zuzusprechen, von deren hier nachgewiesenen gemeinsamen Merkmalen sie z. T. erheblich abweichen.

${ }^{29}$ Bei der nur fragmentarisch angelegten Zeichnung am rechten Rand des Blattes in Besançon könnte es sich scheinend sowohl in detailgenauer Beschreibung des disegno als auch unter Beachtung des malerischen Gesamteindruckes wieder ${ }^{29}$.

Nach den konkreten Spuren einer Auseinandersetzung mit gerade dieser Fassadendekoration Polidoros und Maturinos sucht man unter den Gemälden Poussins indes vergeblich: anders als im Fall der Fassadendekorationen Polidoros und $\mathrm{Ma}$ turinos am direkt gegenübergelegenen Palazzo

ebenfalls um die Rezeption einer Figur Polidoros handeln: Ravelli 1978 (Anm. 22), 138, No. 90 zeigt den Entwurf für eine Fassadendekoration von der Hand Polidoros, deren linke weibliche Karyatide in einzelnen Details (bis hin zu der erhobenen, scherenartig geöffneten Hand) Parallelen zu der Skizze in Besançon zeigt, bei deren Anfertigung vielleicht ein ähnliches Modell vorlag.

${ }^{30}$ Vgl. dazu Pericoli 1960 (Anm. 22), 38-4I; Marabottini 1969 (Anm. 19), Bd. I, I26-129, Bd. 2, 366-369, No. I5; Guide... I978 (Anm. 19), 44f.; Ravelli 1978 (Anm. 22), 367-449, No. 667-9ir.

${ }^{31}$ Vgl. hier: anonymer Zeichner des 17. Jahrhunderts: Kopie der Niobe-Szene am Palazzo Milesi, Städelsches Kunstinstitut, Frankfurt/M., Inv.-No. 542I - vgl. dazu auch Ravelli 1978 (Anm. 22), 367, No. 668/i. Die Niobiden-Szene dieser Fassadendekoration ist heute zusammen mit den übrigen Wandmalereien am Palazzo Milesi - nicht mehr erhalten; sie wurde jedoch - 


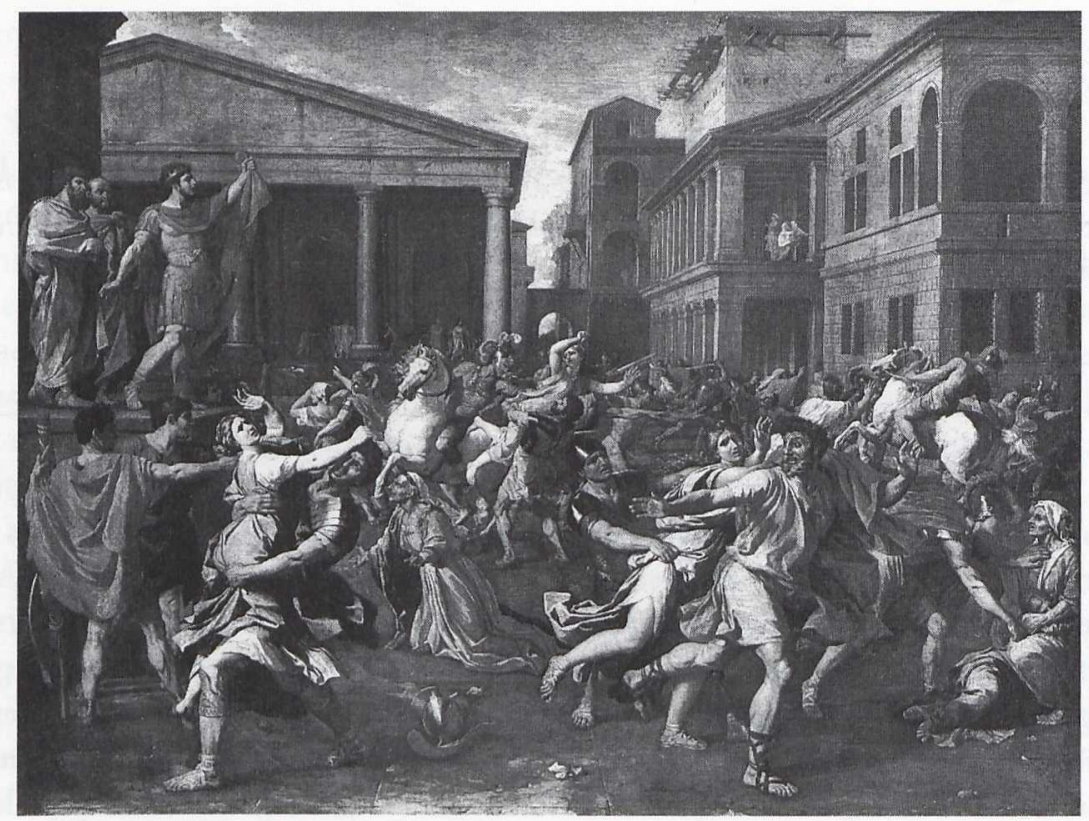

7. Nicolas Poussin, Der Raub der Sabinerinnen, Paris, Musée du Louvre

Milesi $3^{30}$, dessen Niobe-Szene Poussin das Motiv der einem jungen Mädchen Schutz gewährenden Alten (Abb. 6) ) $^{31}$ für seine, gleichfalls in den dreißiger Jahren entstandene Louvre-Fassung ${ }^{32}$ des »Raubes der Sabinerinnen« (Abb. 7) entlehnt hat ${ }^{33}$, scheint er die Karyatiden-Zeichnung eher im Rahmen seiner Suche nach einem neuen, eigenen Zeichenstil angefertigt zu haben. Bewußt oder unbewußt hat er sich damit selbst eine Prüfungsauf-

wie die anderen Elemente und Szenen dieser Malerei - in einer Vielzahl von Zeichnungen und Stichen überliefert; zu diesen vgl. vor allem Ravelli 1978 (s. o. Anm. 30).

${ }^{32}$ Vgl. zur Datierung dieser Fassung zuletzt: Mérot, A.: Poussin, London 1990, 285, No. 187/188.

${ }_{33}$ Dieses Motiv - auf dem Fassadenbild wie im Gemälde an den rechten Bildrand geschoben - findet sich auf zwei Entwurfszeichnungen Poussins (Chatsworth 86r und Uffizien 90oe) zunächst noch im Zentrum des Bildvordergrundes. Zu diesen Zeichnungen vgl. Blunt/ gabe gestellt, die Federico Zuccari und Romano Alberti in ihrer Schrift über die Accademia del Disegno als Bedingung für die Aufnahme eines Schülers in die Akademie vorgeschlagen hatten: jeder Schüler solle eine Zeichnung liefern, »ritratto da qualche opera de gli valent'huomini passati e da quelle particolarmente, che stanno in pericolo di perdersi, e dal tempo annullarsi, di Polidoro, e d'altri valent'huomini... $\ll^{34}$.

Friedländer 1949 (Anm. I0), 9, No. II4/II5. Interessanterweise zeigte ein Bildfeld schräg links über dem Niobe-Fries am Palazzo Milesi auch eine Szene mit dem "Raub der Sabinerinnen « - vgl. dazu den Stich E. Maccaris aus dessen "Graffite e chiaroscuri esistenti all'esterno delle case di Roma «, Rom s.a., abgebildet auch bei Ravelli 1978 (Anm. 22), 369, Abb. 667.

${ }^{34}$ Alberti, R./Zuccari, F.: Origine, et Progresso dell'Academia del Disegno... di Roma, Pavia 1604, 7 (Nachdruck in der Reihe »Italica Gens - Repertori di bio-bibliografia italiana«, No. 96, 1978).

Bildnachweis: I Besançon, Musée des Beaux-Arts et d'Archéologie. - 2 Wien, Bildarchiv der Österreichischen Nationalbibliothek (Neg. ALB 53.57I). - 3 Photographische Reproduktion nach Ravelli, L.: Polidoro Caldara da Caravaggio, Bergamo 1978, 348, No. 614. - 4 The Metropolitan Museum of Art, Gift of Cornelius Vanderbilt, 1880 (80.3.583). - 5 Sopraintendenza Archeologica di Roma (Neg. I87 858/59 E). - 6 Frankfurt a. M., Städel, Inv. 542I (Polidoro da Caravaggio). - 7 Paris, Arts Graphiques de la Cité (AGRACI) 